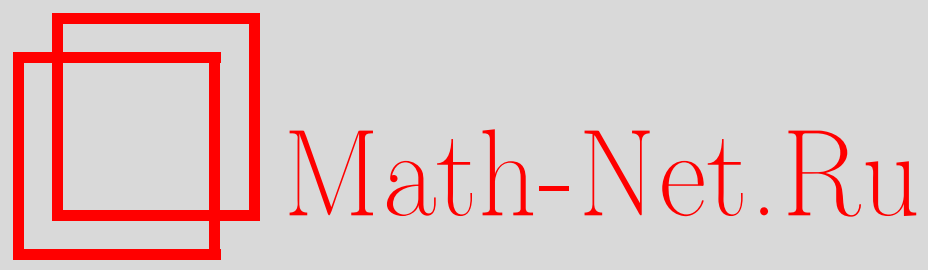

О. В. Шварцман, Кручение в факторгруппе группы кос Артина-Брискорна по центру и регулярные числа Спрингера, Функи. анализ и его прил., 1996, том 30, выпуск 1, 39-46

DOI: https://doi.org/10.4213/faa502

Использование Общероссийского математического портала MathNet.Ru подразумевает, что вы прочитали и согласны с пользовательским соглашением

http://www.mathnet.ru/rus/agreement

Параметры загрузки:

IP : 54.172 .240 .79

26 апреля 2023 г., 13:12:24

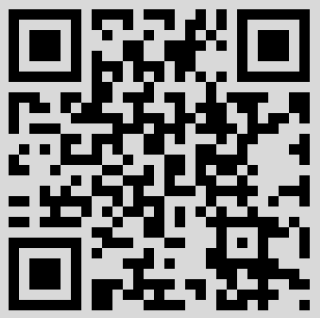




\title{
Кручение в факторгруппе группы кос Артина-Брискорна по центру и регулярные числа Спрингера
}

\author{
(c) 1996 . \\ О. В. ШВАРцМАН ${ }^{1}$
}

Цель статьи - выяснить топологический смысл группы кос АртинаБрискорна, профакторизованной по центру, и найти порядки элементов кручения в указанной факторгруппе. Теорема Мурасуги о кручении в факторгруппе $B(n) / Z(B(n))$ группы кос Артина $B(n)$ по центру $Z(B(n))$ является частным случаем наших результатов при $n \geqslant 5$.

\section{§1. Определения, обозначения, факты}

1.1. На протяжении всей статьи $V$ будет обозначать комплексное векторное пространство, $P=P(V)$ - ассоциированное проективное пространство и $\pi: V-\{0\} \rightarrow P-$ естественную проекцию.

В ситуации когда группа $\Gamma$ действует на пространстве $X$, множество точек $x \in X$ с нетривиальным стабилизатором $\Gamma_{x}$ будет обозначаться через $\operatorname{Fix}(\Gamma, X)$ или просто Fix $\Gamma$, если из контекста ясно, о каком пространстве $X$ идет речь. Для группы линейных преобразований $\Gamma$ пространства $V$ соответствующая группа проективных преобразований пространства $P$ обозначается через $P \Gamma$. Проективизацию линейного преобразования $\gamma$ мы тоже будем обозначать через $P(\gamma)$. Группа $P \Gamma$ изоморфна факторгруппе группы $\Gamma$ по центру $Z(\Gamma)$.

1.2. Линейное преобразование $\gamma$ пространства $V$ называется отражением, если его порядок конечен и $\operatorname{codim}_{\mathbb{C}} \operatorname{Fix} \gamma=1$.

Гиперплоскость неподвижных точек отражения $\gamma$ будем называть его зеркалом. Пусть $W$ - конечная линейная группа, порожденная отражениями, действующая в $r$-мерном комплексном векторном пространстве $V$. Факторпространство $V / W$ изоморфно комплексному векторному пространству $\mathbb{C}^{r}$. Каноническая проекция $\varphi: V \rightarrow V / W$ записывается в координатах формулами

$$
y_{i}=P_{i}\left(x_{1}, \ldots, x_{r}\right), \quad i=1, \ldots, r .
$$

Однородные многочлены $P_{i}\left(x_{1}, \ldots, x_{r}\right)$ степеней $m_{i}(i=1, \ldots, r)$, участвующие в этих формулах, свободно порождают алгебру инвариантов $\mathbb{C}[V]^{W}[1]$.

В дальнейшем, специально этого не оговаривая, мы сохраняем обозначение $\varphi$ и для сужений проекции $\varphi$ на различные объекты в $V$. Пусть $A$ - объединение в $V$ зеркал всех отражений из группы $W$. Вне связки гиперплоскостей $A$

${ }^{1}$ Работа выполнена при финансовой поддержке Американского математического общества. 
группа $W$ действует свободно, т.е. Fix $W=A$. Обозначим через $Y$ факторпространство $(V-A) / W$. Отметим, что $Y$ - комплексное многообразие.

Группа $W$ называется группой Кокстера или группой, порожденной вещественными отражениями, если векторное пространство $V$ является комплексификацией вещественного векторного пространства $V(\mathbb{R})$, в котором действует группа $W$.

1.3. ОПРЕДЕЛЕНИЕ 1 . Вектор $x \in V$ называется $W$-регулярнылм, если $x \notin$ Fix $W$.

ОПРЕДЕлЕНИЕ 2. Элемент $w \in W$ называется регулярныл, если существует $W$-регулярный вектор $x$, собственный для линейного преобразования $w$.

Рассмотрим множество $\operatorname{Ord}\left(W_{\text {reg }}\right)$, состоящее из порядков всех регулярных элементов группы $W$. Вместе с любым порядком в это множество входят и все его делители.

ОПРеДЕлЕнИЕ 3. Регулярным набором Спрингера $\left(r_{1}, \ldots, r_{m}\right)$ называется множество максимальных элементов в $\operatorname{Ord}\left(W_{\text {reg }}\right)$ по отношению делимости [13].

Переходя к группе $P W$, естественно определить модифииированный регулярный набор Спрингера. Такой набор $\left(\hat{r}_{1}, \ldots, \hat{r}_{m}\right)$ получается из исходного по правилу

$$
\hat{r}_{i}=r_{i} /\left(|Z(W)|, r_{i}\right)
$$

(используются стандартные обозначения: $(a, b)$ - для НОД $(a, b)$ и $|Z|-$ для числа элементов в множестве $Z$ ).

1.4. Фундаментальная группа $\pi_{1}(Y)$ (соответственно фундаментальная группа $\left.\pi_{1}(V-A)\right)$ пространства $Y$ называется группой обобщенных кос Apmuна-Брискорна (соответственно группой обобщенных крашеных кос) и обозначается $B(W)$ (соответственно $C(W)$ ). В дальнейшем мы будем говорить просто о әруппе кос $B(W)$ или группе крашеньх кос $C(W)$. Группа крашеных кос является нормальной подгруппой группы кос. Отметим, что в группе кос нет кручения [2].

\section{§2. Результаты}

Пусть $G$ - конечная группа проективных преобразований пространства $P$, порожденная отражениями. Среди действующих в пространстве $V$ конечных линейных групп $\Gamma$, порожденных отражениями и таких, что $P \Gamma=G$, выберем единственную максимальную (по включению) группу $W$ [12]. Введем в рассмотрение следующие пространства:

$$
\widehat{A}=\pi(A), \quad \widehat{P}=P-\operatorname{Fix}(G, P), \quad \widehat{V}=\pi^{-1}(\widehat{P}), \quad \widehat{X}=\widehat{P} / G, \quad \widehat{Y}=\widehat{V} / W .
$$

Предположим, что $W$ - неприводимая группа Кокстера в пространстве $V$. В этом случае будут доказаны две теоремы.

Теорема А. Фундаментальная группа $\pi_{1}(\widehat{X})$ пространства $\widehat{X}$ изомор $\phi-$ на apynne $B(W) / Z(B(W))$.

Теорема В. Пусть $\gamma$ - элемент конечного порлдка $t$ в группе $\pi_{1}(\widehat{X})$. Тогда $t$ делит одно из чисел модифичированного набора Спрингера группь $W$. Обратно, для любого делителя $t$ какого-либо из чисел $\hat{r}_{1}, \ldots, \hat{r}_{m}$ существует элемент $\gamma \in \pi_{1}(\widehat{X})$, порлдок которого равен $t$. 
СлЕДСТвИЕ [10]. При $n \geqslant 5$ в группе $B(n) / Z(B(n))$ әлемент данного конечного порядка $t$ существует тогда и только тогда, когда $t$ делит одно из чисел $n-1, n$.

\section{§3. Доказательство}

3.1. Для доказательства теоремы А требуются приготовления.

Лемма 1. Фундаментальные группь пространств $Y$ и $\widehat{Y}$ совпадают.

ДокАЗАтЕльство. Пространство $\widehat{Y}$ получается из многообразия $Y$ удалением аналитического подмножества $\pi^{-1}(\operatorname{Fix}(G, P-\widehat{A})) / W$ комплексной коразмерности $\geqslant 2$ (здесь мы воспользовались максимальностью группы $W$ ). В таком случае, согласно известному топологическому факту, фундаментальные группы $\pi_{1}(\widehat{Y})$ и $\pi_{1}(Y)$ совпадают. Лемма доказана.

Совершенно по тем же причинам совпадают и фундаментальные группы пространств $V-A$ и $\widehat{V}$.

Лемма 2. Пространство $P-\widehat{A}$ является пространством типа $K(\pi, 1)$.

ДокАЗАТЕЛЬСтво. Воспользуемся тем фактом, что пространство $V-A$, а следовательно, и пространство $Y$ являются пространствами типа $K(\pi, 1)[4,7]$. Легко показать, что у естественного $\mathbb{C}^{*}$-расслоения $\pi: V-A \rightarrow P-\widehat{A}$ есть сечение. Следовательно, для всех $i \geqslant 2$ имеется разложение в прямую сумму (см. [3]) $\pi_{i}(V-A)=\pi_{i}(P-\widehat{A}) \oplus \pi_{i}\left(\mathbb{C}^{*}\right)$. Поскольку у $\mathbb{C}^{*}$ все гомотопические группы, начиная со второй, тривиальны, то лемма 2 доказана.

Вернемся к $\mathbb{C}^{*}$-расслоению $\pi: V-A \rightarrow P-\widehat{A}$ и запишем с учетом результата леммы 2 фрагмент точной гомотопической последовательности расслоения:

$$
1 \rightarrow \pi_{1}\left(\mathbb{C}^{*}\right) \stackrel{\mu}{\rightarrow} \pi_{1}(V-A) \rightarrow \pi_{1}(P-\widehat{A}) \rightarrow 1 .
$$

Наша ближайшая цель - вычислить образ группы $Z$ (фундаментальной группы слоя) при ее вложении $\mu$ в группу $C(W)=\pi_{1}(V-A)$ (а значит, и в группу $B(W))$. Но прежде, чем следовать дальше, уместно сделать

ЗАмЕЧАНИЕ 1 . Если центр группы $W$ изоморфен $\mathbb{Z}_{2}$, то центр группы кос $Z(B(W))$ - это циклическая группа $\langle\Delta\rangle$, порожденная фундаментальным элементом $\Delta$. Если же центр группы $W$ тривиален, то центр $Z(B(W))$ совпадает с циклической группой $\left\langle\Delta^{2}\right\rangle[6,7]$. В любом случае элемент $\Delta^{2}$ лежит в центре группы кос.

Лемма 3. Образ $\mu\left(\pi_{1}\left(\mathbb{C}^{*}\right)\right)$ в группе $C(W)$ coвпадает с ииклической подәруппой $\left\langle\Delta^{2}\right\rangle$.

ДокАЗАТЕЛЬСТво. Пусть $H$ - зеркало вещественного отражения $w$ из группы $W$. Выберем в пространстве $V(\mathbb{R})$ точку $Q \notin H$ и рассмотрим комплексную прямую $L$ в $V$, соединяющую точки $Q$ и $Q^{\prime}=w(Q)$. Обозначим через $l\left(Q, Q^{\prime}\right)$ путь в $V$, состоящий из направленного вещественного отрезка $Q Q^{\prime}$, у которого «маленькая» $\varepsilon$-окрестность точки $L \cap H$ заменена «верхней» полуокружностью $R^{+}$радиуса $\varepsilon$, лежащей в $L$. Элемент $\sigma$ фундаментальной группы $\pi_{1}(\varphi(Q), Y)$, соответствуюший пути $l\left(Q, Q^{\prime}\right)$, назовем $w$-стандартным. 
Пусть $K$ - фундаментальная камера Вейля группы $W$ в пространстве $V(\mathbb{R})$, a $S=\left\{w_{1}, \ldots, w_{r}\right\}$ - порождающие эту группу отражения в гиперплоскостях, ограничивающих камеру $K$. Зафиксируем внутри камеры $K$ точку $Q_{0}$. Coгласно Брискорну, $w_{i}$-стандартные элементы $\sigma_{i}$ порождают фундаментальную группу $\pi_{1}\left(\varphi\left(Q_{0}\right), Y\right)$, изоморфную группе кос $B(W)$ [5]. Рассмотрим в группе $(W, S)$ элемент $\bar{\Delta}$ максимальной длины $q[1]$. Пусть $\bar{\Delta}=\prod_{i=1}^{q} w_{s_{i}}-$ запись элемента $\bar{\Delta}$ через образующие $w_{1}, \ldots, w_{r}$. Тогда фундаментальный элемент $\Delta$ записывается с помощью образующих $\sigma_{1}, \ldots, \sigma_{r}$ словом $\prod_{i=1}^{q} \sigma_{s_{i}}[6-8]$. Удобно ввести элементы $\bar{\Delta}_{j}=\prod_{i=1}^{j} w_{s_{i}}(j=1, \ldots, q)$ в группе $W$ и соответственно элементы $\Delta_{j}=\prod_{i=1}^{j} \sigma_{s_{i}}$ в группе кос.

Известно, что $\bar{\Delta}(K)=-K$. Соединим камеры $K$ и $-K$ цепочкой смежных камер $K, \bar{\Delta}_{1}(K), \ldots, \bar{\Delta}_{q} K=-K\left(\bar{\Delta}_{0}=\right.$ id и $\left.\bar{\Delta}_{q}=\bar{\Delta}\right)$. Переход из камеры $\bar{\Delta}_{j-1} K$ в смежную камеру $\bar{\Delta}_{j}(K)$ осуществляется с помощью отражения $\bar{\Delta}_{j-1} w_{s_{j}} \bar{\Delta}_{j-1}^{-1}$ в их общей грани $H_{j}$. Но множество гиперплоскостей $\left\{H_{j}\right\}$ - это множество зеркал всех отражений из группы $W[1]$. Поэтому в множестве всех зеркал $A$ возникает естественный порядок $H_{1}<\cdots<H_{q}$.

Далее, рассмотрим точки $Q_{j}=\bar{\Delta}_{j}\left(Q_{0}\right)$ и кривые $l_{j}=l\left(Q_{j-1}, Q_{j}\right)(j=$ $1, \ldots, q)$. Кривая $l_{j}$ представляет в фундаментальной группе $\pi_{1}\left(\varphi\left(Q_{0}\right), Y\right)$ стандартную образуюшую $\sigma_{s_{j}}$. Совершим в пространстве $V-A$ обход $O_{j}$ гиперплоскости $H_{j}$ по следующему правилу: из точки $Q_{0}$ проходим до точки $Q_{j}$, двигаясь по цепочке кривых $l_{1}, \ldots, l_{j}$, затем проходим путь $l_{j}$ в обратном направлении с той лишь разницей, что точку $H_{j} \cap L_{j}$ обходим в $L_{j}$ по «нижней» полуокружности $R_{j}^{-}$, после чего из точки $Q_{j-1}$ возвращаемся в $Q_{0}$, проходя прежний путь в обратном направлении. Прямое вычисление показывает, что построенный обход гиперплоскости $H_{j}$ соответствует элементу $\alpha_{j}=\Delta_{j-1} \sigma_{s_{j}}^{2} \Delta_{j-1}^{-1}$ Фундаментальной группы $\pi_{1}\left(\varphi\left(Q_{0}\right), Y\right)$, а произведение всех обходов $\prod_{j=1}^{q} O_{j}$ — элементу $\alpha=\sigma_{s_{1}} \cdots \sigma_{s_{q}} \sigma_{s_{q}} \cdots \sigma_{s_{1}}$. Ввиду того что $\sigma_{s_{1}} \cdots \sigma_{s_{q}}=\sigma_{s_{q}} \cdots \sigma_{s_{1}}$ ([6, лемма 5.1]), $\alpha=\Delta^{2}$.

Воспользуемся, наконец, стандартными аргументами Зариского, сведя все к случаю пучка прямых в $\mathbb{C}^{2}$ (см., например, [11]). Подробнее: за счет выбора точки $Q_{0}$ (в силу замечания 1 утверждение леммы от этого выбора не зависит) добьемся того, чтобы вещественный отрезок $\left[Q_{0}, \bar{\Delta}\left(Q_{0}\right)\right]$ пересекал лишь грани коразмерности 1 симплициального разбиения пространства $V(\mathbb{R})$ камерами группы $W$. Затем рассмотрим комплексную прямую $l$, проходящую через точки $Q_{0}$ и $\bar{\Delta}\left(Q_{0}\right)$, и заменим каждый путь $O_{j}$ гомотопным ему путем $O_{j}^{\prime}$, лежащим в $l$ (это можно сделать, очевидным образом стягивая «вещественную” часть пути $O_{j}$ внутри каждой камеры на часть вещественного отрезка $\left[Q_{0}, \bar{\Delta}\left(Q_{0}\right)\right]$, которая лежит в этой камере). Одновременно позаботимся и о том, чтобы пути $Q_{j}^{\prime}$ не имели других общих точек, кроме точки $Q_{0}$. Теперь мы рассмотрим комплексную плоскость $V^{\prime}$ в пространстве $V$, содержашую прямую $l$, и индуцированный на ней связкой $A$ пучок прямых $\left\{m_{j}^{\prime}\right\}=V^{\prime} \cap A$. Известно, что произведение $O^{\prime}=\prod_{j=1}^{q} O_{j}^{\prime}$ построенных нами обходов $O_{j}^{\prime}$ вокруг точек $c_{j}^{\prime}=m_{j}^{\prime} \cap l$ служит образующей фундаментальной группы слоя в $\mathbb{C}^{*}$-расслоении 
$V^{\prime}-\left\{m_{j}^{\prime}\right\} \rightarrow l-\left\{c_{j}^{\prime}\right\}$ (см., например, [11]). А так как $\alpha=O^{\prime}$ по построению, то лемма 3 доказана.

Бесхитростной проверкой определений с помощью формулы (1) доказывается

Лемма 4. Регулярное неразветвленное накрытие $\varphi: \widehat{V} \rightarrow \widehat{Y}$ является морфизмом главных $\mathbb{C}^{*}$-расслоений $\pi: \widehat{V} \rightarrow \widehat{P}$ u $\widehat{\pi}: \widehat{Y} \rightarrow \widehat{X}$.

Действие группьл $\mathbb{C}^{*}$ на пространстве $Y$ задаетсл в координатах $y_{1}, \ldots$, $y_{r}$ (см. п. 1.2) формулой

$$
t\left(y_{1}, \ldots, y_{r}\right)=\left(t^{m_{1}} y_{1}, \ldots, t^{m_{r}} y_{r}\right), \quad t \in \mathbb{C}^{*} .
$$

На слоях отображение $\varphi$ является регулярным неразветвленным накрытием $\mathbb{C}^{*} \rightarrow \mathbb{C}^{*}$ степени, равной $|Z(W)|$. Объяснить это можно так: по определению пространства $\widehat{V}$ никакой вектор из $\widehat{V}$ не является собственным вектором элемента из группы $W$, отличного от элемента центра.

Запишем фрагмент коммутативной диаграммы точных гомотопических последовательностей расслоений, связанной с морфизмом $\varphi$ :

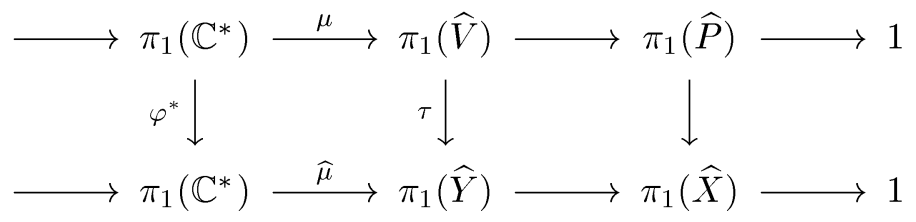

Отметим, что отображения $\varphi^{*}$ и $\tau$ являются мономорфизмами.

Если центр группы $W$ тривиален, то $\varphi^{*}$ - изоморфизм, а если порядок центра равен 2 , то образ отображения $\varphi^{*}$ - подгруппа индекса 2 . В первом случае диаграмма (2) вместе с результатом леммы 3 показывают, что образ $\widehat{\mu}\left(\pi_{1}\left(\mathbb{C}^{*}\right)\right)$ в группе $\pi_{1}(\widehat{Y})=B(W)$ совпадает с подгруппой $\left\langle\Delta^{2}\right\rangle$, а во втором — что этот образ порождается таким элементом $\delta \in B(W)$, что $\delta^{2}=\Delta^{2}$. Но так как при этом элемент $\Delta$ лежит в центре группы $B(W)$ (см. замечание 1$)$, то $\delta=\Delta$. В противном случае мы обнаружили бы 2-кручение в группе $B(W)$, свободной от кручения. Итак, в обоих случаях фундаментальная группа $\pi_{1}(\widehat{X})$ изоморфна группе $B(W) / Z(B(W))$.

3.2. Доказательство теоремы В удобно разбить на две части. Сначала рассмотрим общую ситуацию, когда на связном гладком многообразии $M$ дискретно действует группа автоморфизмов Г. Пусть $\psi: M \rightarrow M / \Gamma$ - естественное отображение и $p: \widetilde{M} \rightarrow M-$ универсальное накрытие многообразия $M$. Обозначим через $\widetilde{\Gamma}$ группу автоморфизмов универсальной накрывающей $\widetilde{M}$, состоящую из всевозможных подъемов $\widetilde{\gamma}$ элементов $\gamma$ из Г. Отождествляя фундаментальную группу $\pi_{1}(M)$ с группой скольжений универсального накрытия, получаем точную последовательность групп $1 \rightarrow \pi_{1}(M) \rightarrow \widetilde{\Gamma} \stackrel{\widetilde{p}}{\rightarrow} \Gamma \rightarrow 1$ (см., например, [9]).

ЛЕмма 5. Для любой точки $\tilde{x} \in \widetilde{M}$ каноническал проекчия $\widetilde{p}$ изоморфно отображает стабилизатор $\widetilde{\Gamma}_{\tilde{x}}$ точки $\tilde{x}$ на стабилизатор $\Gamma_{x}$ точки $x=$ $p(\tilde{x}) \in M$. 
ДоКАЗАТЕЛЬСТВо. Подъем $\widetilde{\gamma}$ любого элемента $\gamma \in \Gamma$ определяется однозначно, если указать образ фиксированной точки $\widetilde{x} \in \widetilde{M}$ при действии $\widetilde{\gamma}$, причем этим образом может служить любая точка слоя $p^{-1}(\gamma(p(\tilde{x})))$. По этой причине стабилизатор точки $x \in M$ вкладывается в стабилизатор любой точки $\tilde{x} \in p^{-1}(x)$. Принимая во внимание, что $\widetilde{p}\left(\widetilde{\Gamma}_{\widetilde{x}}\right) \subset \Gamma_{x}$, получаем утверждение леммы.

Перед тем как доказывать ключевую лемму 6 , договоримся через $|g|$ обозначать порядок элемента группы.

ЛЕмма 6. Предположим, что гладкое многообразие $M$ и дискретная группа его автоморфизмов Г обладают следующими дополнительными свойствами:

(a) $M$ - пространство типа $K(\pi, 1)$;

(b) любой элемент конечного порлдка из группь $Г$ имеет неподвижную точку при действии на $M$;

(c) $\operatorname{codim}_{\mathbb{R}} \operatorname{Fix}(\Gamma, M) \geqslant 3$.

\section{Тогда}

1. Если $\widetilde{\gamma} \in \widetilde{\Gamma}$ - элемент конечного порядка и $\gamma=\widetilde{p}(\widetilde{\gamma})$, то $|\gamma|=|\widetilde{\gamma}|$.

2. Для любого элемента конечного порядка $\gamma \in \Gamma$ найдется такой элемент $\widetilde{\gamma} \in \widetilde{\Gamma}$, ито $\widetilde{p}(\widetilde{\gamma})=\gamma$ u $|\widetilde{\gamma}|=|\gamma|$.

3. Фундаментальная группа пространства $M / \Gamma-\psi($ Fix $Г)$ изоморфна apynne $\widetilde{\Gamma}$.

ДокАЗАТЕЛЬСтво. 1. Пусть $\widetilde{\gamma}$ - элемент конечного порядка из группы $\widetilde{\Gamma}$. Тогда $\widetilde{\gamma}$ имеет неподвижную точку $\tilde{x}$ при действии на $\widetilde{M}$, поскольку $M-$ пространство типа $K(\pi, 1)[3]$. Следовательно, элемент $\gamma=\widetilde{p}(\widetilde{\gamma})$ из группы $Г$ оставляет неподвижной точку $x$, и $|\widetilde{\gamma}|=|\gamma|$ по лемме 5 .

2. Если $\gamma$ - элемент конечного порядка из $\Gamma$ и $x$ - его неподвижная точка в $M$, то для любой точки $\tilde{x} \in p^{-1}(x)$ в стабилизаторе $\widetilde{\Gamma}_{\tilde{x}}$ есть элемент $\widetilde{\gamma}$ порядка $|\gamma|$ (снова лемма 5).

3. Еще раз воспользуемся тем фактом, что многообразие $\widetilde{M}$ остается односвязным, если из него удалить подмногообразие $p^{-1}(\operatorname{Fix}(\Gamma, M))$ вещественной коразмерности $\geqslant 3$.

Второй шаг доказательства теоремы В объясняет связь порядков элементов кручения в фундаментальной группе $\pi_{1}(\widehat{X})$ с модифицированным набором Спрингера.

Назовем элемент $g$ группы $G$ регулярнылм, если у него есть неподвижная точка в пространстве $P-\widehat{A}$. Обозначим через $w(g)$ элемент из группы $W$, такой, что $P(w(g))=g$. Ясно, что $w(g)$ является $W$-регулярным элементом.

ЛЕмма 7. Предположим, что $|w(g)|=d$, а порядок иентра группь $W$ равен $m$. Тогда порядок элемента $g$ равен $d /(d, m)$.

ДокаЗАТЕЛЬСТво. Если два элемента $w_{1}$ и $w_{2}$ из $W$ имеют общий регулярный собственный вектор $v \in V$ с одинаковыми собственными значениями, то они равны. В самом деле, тогда элемент $w_{1} w_{2}^{-1}$ имеет неподвижный регулярный вектор $v$ и, следовательно, $w_{1}=w_{2}$. Таким образом, утверждение о порядке элемента $g$ следует из хорошо известных свойств группы корней из единицы. 
ПРЕДЛОЖЕНИЕ 1. Порядки регулярных элементов группь $G$ - это делители модифицированного набора Спрингера группь $G$ и только они.

ДокАЗАТЕЛЬСтво. Пусть $\left\langle r_{1}, \ldots, r_{m}\right\rangle$ - регулярный набор Спрингера группы $W$ и $d$ - делитель некоторого $r_{k}, 1 \leqslant k \leqslant m$. Тогда регулярный элемент порядка $d$ в группе $W$ дает нам по лемме 7 регулярный элемент порядка $d /(d, m)$ в группе $G$. Так как $d /(d, m)$ - делитель числа $r_{k} /\left(r_{k}, m\right)$, то в одну сторону предложение доказано. В обратную сторону: пусть $t$ - делитель некоторого числа $\hat{r}_{k}=r_{k} /\left(r_{k}, m\right)$. Тогда $t \cdot(t, m)$ - делитель $r_{k}$, и регулярный элемент порядка $t \cdot(t, m)$ в группе $W$ имеет порядок $t$ в группе $G$.

Наконец, выбрав в лемме 6 в качестве $M$ пространство $P-\widehat{A}$, в качестве $\Gamma$ - конечную группу $G$ автоморфизмов $K(\pi, 1)$-пространства $P-\widehat{A}$ (лемма 2 !) и сопоставив результаты леммы 6 и предложения 1 , мы докажем теорему В.

3.3. Чтобы доказать следствие, рассмотрим симметрическую группу $S_{n}$ в ее стандартном кокстеровском представлении в пространстве $V$ размерности $n-1$. Так как центр симметрической группы тривиален, то проективная группа $G=P S_{n}$ изоморфна группе $S_{n}$. Изоморфна $S_{n}$ и максимальная группа $W$, но только при $n \geqslant 5$ (по этому поводу см. п. $1 \S 4$ ). Регулярный набор Спрингера группы $S_{n}$ равен $(n-1, n)[13]$. Следовательно, по теоремам А и В при $n \geqslant 5$ порядки элементов кручения в группе $B(n) / Z(B(n))$ - это ровно все делители чисел $n-1$ и $n$, что и требовалось доказать.

\section{§4. Комментарии и гипотеза}

1. Рассматривая классификационные таблицы Шепарда-Тодда [12] неприводимых линейных групп, порожденных отражениями, нетрудно убедиться в следующем.

Пусть $\widetilde{W}$ - неприводимая группа Кокстера в пространстве $V$ размерности $r$. Тогда максимальная линейная группа $W$ (см. $\S 2)$ для группы $G=P \widetilde{W}$ совпадает с $\widetilde{W}$, если $r \geqslant 4$. В размерности 3 единственное исключение представляет группа $S_{4}$ : группа $W$ изоморфна группе Вейля системы корней $B_{3}$. Хуже обстоит дело в размерности 2, где каждый элемент конечного порядка становится отражением в проективной группе. Так, например, для группы $S_{3}$ группа $W$ изоморфна некокстеровской группе № 7 из списка Шепарда-Тодда [12].

По этой причине наш метод доказательства теоремы Мурасуги, к сожалению, не «работает» в размерностях 2 и 3 ( $n=3$ и $n=4$ соответственно).

2. Спрингер вычислил регулярные числа для всех неприводимых конечных линейных групп, порожденных отражениями [13].

3. Кроме порядков элементов кручения в группе $B(n) / Z(B(n))$ в работе [10] найдены канонические представители классов сопряженных элементов конечного порядка. Эту часть результатов Мурасуги при $n \geqslant 5$ несложно получить, скомбинировав результат леммы 5 и три следующих утверждения о действии группы $G$ в пространстве $P-\widehat{A}$, первые два из которых фактически доказаны в [13].

ПредлОЖЕНИЕ 2. Стабилизатор $G_{x}$ точки $x \in P-\widehat{A}$ является ииклической аруппой, порожденной регулярным элементом.

Точка $x \in P-\widehat{A}$ называется $s$-точкой, если $\left|G_{x}\right|=s$. 
ПРЕДЛОЖЕНИЕ 3. Пусть $g$ - регулярный элемент порлдка $d$ в группе $G$. Если d делит некоторое число $\hat{r}_{j}, 1 \leqslant j \leqslant m, u x-$ любая $\hat{r}_{j}$-точка, то әлемент $g$ сопряжен элементу из стабилизатора $G_{x}$.

ПРЕДЛОЖЕНИЕ 4. Если $G=S_{n}$, то все $(n-1)$-точки (соответственно n-точки) образуют одну $S_{n}$-орбиту в $P-\widehat{A}$.

Мы не будем здесь детально рассматривать случай группы $S_{n}$, надеясь в будущем вернуться к вопросу об описании классов сопряженных элементов кручения в общей ситуащии теорем А и В.

4. ГиПоте3А. Теоремы А и В верны без дополнительного предположения о кокстеровости группь $W$.

\section{ЛитеРАТУРА}

1. Бурбаки Н. Группы и алгебры Ли. Мир, М. (1972).

2. Лин В. Я. Косы Артина и связанные с ними группы и пространства. Итоги науки и техники. Алгебра, Геометрия, Топология, т. 17, ВИНИТИ, М. (1979), c. $159-227$.

3. Ху Сы-Цзян. Теория гомотопий. Мир, М. (1964).

4. Brieskorn E. Sur les groupes de tresses (d'apres V. I. Arnold). Lect. Notes Math., Vol. 317 (1973), pp. 21-44.

5. Brieskorn E. Die Fundamentalgruppe des Raumes der regularen Orbits einer endlichen komplexen Spiegelungsgruppe. Invent. Math., 12, 57-61 (1971).

6. Brieskorn E., Saito K. Artin-Gruppen und Coxeter-Gruppen. Invent. Math., 17, 245-271 (1972).

7. Deligne P. Les immeubles des groupes de treeses generalises. Invent. Math., 17, 273-302 (1972).

8. Garside F. The braid group and other groups. Quart. J. Math. Oxford., 20, 235-254 (1969).

9. Maskit B. Kleinian Groups. Springer-Verlag, Berlin-Heidelberg-New York (1988).

10. Murasugi K. Seifert fibre spaces and braid groups. Proc. London Math. Soc., 44, 71-84 (1982).

11. Orlic P., Terao H. Arrangements. Springer-Verlag, Berlin-Heidelberg-New York (1992).

12. Shephard G., Todd J. Finite unitary reflection groups. Can. J. Math., 6, 274-304 (1954).

13. Springer T. Regular elements of finite reflection groups. Invent. Math., 25, 159-198 (1974).

Московский технический университет

Поступило в редакцию связи и информатики

15 июня 1994 г.

Московский независимый университет 